\title{
A solved form algorithm for ask and tell Herbrand constraints *
}

\author{
Maurizio Gabbrielli Giorgio Levi \\ Dipartimento di Informatica \\ Università di Pisa \\ Corso Italia 40, 56125 Pisa, Italy \\ e.mail: \{gabbri,levi\}@dipisa.di.unipi.it
}

\begin{abstract}
We consider the problem of defining a solved form for ask and tell equalities over the Herbrand universe used as semantic domain of concurrent constraint programs. We give a solved form which is unique up to renaming for equivalent constraints, and we show an algorithm to compute it. The solved form and the related algorithm are extended to reactive trees consisting of ask and tell constraints. The algorithms are finally shown to be correct, i.e. to preserve the semantics of ask and tell constraints and of reactive trees.
\end{abstract}

\section{Introduction}

The Constraint Logic Programming (CLP) paradigm [13] is a powerful and elegant extension of logic programming. The basic idea is to replace term unification by constraint satisfaction over specific domains. Standard logic programming is just a special instance of CLP, where the constraints are equalities over the Herbrand universe (the resulting constraint system will be called H).

Constraint satisfaction (including its instance on $\mathcal{H}$, i.e. unification) can in principle be splitted into two separate algorithms. The first algorithm deals with consistency checking. Both the operational and the algebraic semantics $[14,13,9]$ can be defined by using consistency checking only. As a matter of fact, a CLP computation can simply be viewed as a process which incrementally accumulates consistent sets of constraints.

The second algorithm deals with the generation of the solutions of the set of constraints. In the case of $\mathcal{H}$, this is a transformation algorithm which reduces a set of equations to an equivalent set in solved form $[21,16]$. Similar algorithms have been proposed for other constraint systems, namely equalities and inequalities over the Herbrand universe with infinite functors [15,3] and generalized linear arithmetic constraints [17]. Solved form algorithms are practically very important from the computational viewpoint. In addition, they sometimes define a kind of normal form for constraints. Namely two equivalent constraints have the same solved form (possibly modulo simple syntactic equivalence relations such as variance).

The power of the CLP paradigm was further extended by the introduction of the notion of ask constraint [20]. Its aim was modeling, in a purely logic-algebraic setting, the typical synchronization

\footnotetext{
*This work was supported by ESPRIT Basic Research Action P3020 "Integration" and by C.N.R. Progetio Finalizzato "Sistemi Informatici e Calcolo Parallelo"
} 
mechanisms of concurrent logic languages [26]. The semantics of ask constraints can informally be described as follows. If $\sigma$ is the current state (a consistent set of constraints) and $\alpha$ is an ask constraint to be evaluated in $\sigma$, the evaluation of $\alpha$ succeeds if $\sigma$ entails $\alpha$. Note that, in the case of a standard constraint $\tau$, the evaluation of $\tau$ in $\sigma$ succeeds if $\sigma U \tau$ is consistent (standard constraints are called tell constraints when needed to distinguish them from the ask constraints). The evaluation of an ask constraint $\alpha$ in the state $\sigma$ fails if $\sigma \cup \alpha$ is inconsistent (this is true for tell constraints as well). The most interesting case, which applies to ask constraints only and characterizes synchronization, is the one in which $\sigma \cup \alpha$ is consistent but $\sigma$ does not entail $\alpha$. In such a case, the evaluation of $\alpha$ suspends and can possibly be resumed if $\sigma$ is changed to a new state entailing $\alpha$.

The presence of ask constraints leads to the violation of some important semantic properties of logic programs (and of constraint logic programs). Namely the lifting lemma [19,1] does not hold any more, i.e. an atomic goal $G$ can fail even if an instance of $G$ succeeds. As a consequence the result of the computation is not independent from the selection rule, i.e. the order in which the atoms in a conjunctive goal are evaluated can affect the computation result.

The structure of a concurrent constraint clause is the following [24]

$$
\text { c } H:- \text { ask }: \text { tell } \rightarrow A_{1}, \ldots, A_{n}
$$

where $H, A_{1}, \ldots, A_{n}$ are atoms and $a s k$, tell are constraints. Let us consider an atomic goal $G$ in the state $\sigma$ ( $G$ and $c$ do not share variables). If $\sigma^{\prime} \equiv \sigma \cup\{H=G\} \cup a s k \cup$ tell is consistent, and $\sigma \cup\{H=G\}$ entails ask, the computation of $G$ can commit to clause $c(\rightarrow$ is the commit operator), by nondeterministically choosing $c$ among the other clauses applicable to $G$. As a consequence, the new goal is $A_{1}, \ldots, A_{n}$ and the new state is $\sigma^{\prime}$.

It has been shown $[23,18,8,4]$ that because of synchronization (and of the related dependency from the selection rule), at least sequences of ask and tell constraints are needed to describe the success set semantics of concurrent logic programs. If the semantics has to model finite failures and deadlocks, ask and tell constraints can be arranged into tree structures [25,10]. More abstract semantics based on sequences have been defined. In [11] sequences of ask and tell constraints are enriched with failure set information. Recent results $[5,7,6]$ show that sequences of assume and tell constraints are also adequate to model finite failures and deadlocks (but assume constraints are different from ask constraints). Since we are interested in generalizing the formal setting of logic programming and CLP, where the syntactic objects (atoms and constraints) are also the basic ingredients of the semantic domains, we will be concerned with ask and tell trees (reactive trees). Of course suitable equivalence relations on reactive trees have to be defined in order to get a more abstract semantics. One basic component of these relations, concerned with the structure of the actions, is an equivalence relation on ask and tell constraints, possibly defined by a transformation to normal form.

In this paper we are concerned with the problem of defining such a transformation in the case of ask and tell constraints over the constraint system $\mathcal{H}$. This case is particularly interesting, because very closely resembles flat concurrent logic languages [26]. In the $\mathcal{H}$ constraint system, constraints are equations. As we will discuss in the next section, ask equations can be represented by constraints using a different predicate ( $\leq$, one-way unification), originally proposed in PARLOG [2].

The objects we are interested in are formulas (reactive trees composed of $\leq$ and = constraints), whose semantics can be understood in terms of consistency only. We will show that $\leq$ and $=$ constraints have a solved form which preserves the semantics and which is unique up to variables renaming for equivalent constraint. The solved form of $\leq$ and $=$ constraints is then extended to reactive trees. An algorithm is given which transforms reactive trees to their solved forms. The algorithm is finally shown to be correct. 


\section{One-way unification constraints}

As already mentioned, in ask and tell languages over the constraint system $\mathcal{H}$, tell constraints are simply unification atoms, while the ask mechanism can be "implemented" by a suitable notion of restricted unification. In particular, as shown in [8], PARLOG one-way unification because of its "local" nature, can fully represent the logical ask mechanism of concurrent constraint languages over $\mathcal{H}$.

The one-way unification primitive was defined in $[2,12]$ as follows.

Definition 2.1 (one-way unification $(\leq)$ ) Let $s$ and $t$ be two first order terms.

i) If there exists an idempotent mgu $\theta$ of $s$ and $t$ such that $\theta$ does not bind variables in $s$ then $s \leq t$ succeeds with c.a.s. $\theta$.

ii) If $s$ and $t$ can be unified only by binding variables in $s$, then $s \leq t$ suspends.

iii) If $s$ and $t$ cannot be unified $s \leq t$ fails.

We will just consider a simple example.

\section{Example 2.2}

c) $s(X, Y):-\{Y=f(Z), X=a\}:\{Z=g(W)\} \rightarrow t(b, W)$.

$\left.c_{2}\right) t(X, Y):-\{X=b\}:\{Y=a\}$.

According to the ask evaluation rule mentioned in the previous section, the goal $s(a, f(X))$ succeeds computing the answer constraint $X=g(a)$, while the (more general) goal $s(X, Y)$ leads to a deadlock. The same results can be obtained in the following program, where the ask's are realized by one-way unifications.

c) $s(X, Y):-Y \leq f(Z), X \leq a: Z=g(W) \rightarrow t(b, W)$.

$\left.c_{2}\right) t(X, Y):-X \leq b: Y=a$.

\section{Reactive trees}

In this section we give the basic definitions of reactive sequences and reactive trees, together with their intended semantics. Informally, a reactive tree is an $\mathrm{OR}$ tree, rooted in a predicate definition, whose edges are labeled by ask and tell constraints. Tell and ask elements can be considered as actions and reactions performed by the agent (the root predicate) and by its external environment along the computation. In the following $\operatorname{Var}(t)$ where $t$ is a term, a sequence or a set of terms, denotes the set of variables in $t$. Reactive trees and reactive sequences are defined as follows.

Definition 3.1 (reactive tree)

$$
\begin{aligned}
& \text { Tree }::=\text { Head }:- \text { Agent } \quad \text { Head }::=p\left(X_{1}, \ldots, X_{n}\right) \\
& \text { ask }::=\left\{s_{1} \leq t_{1}, \ldots, s_{n} \leq t_{n}\right\} \text { tell }::=\left\{s_{1}=t_{1}, \ldots, s_{n}=t_{n}\right\} \\
& \text { Agent }::=\text { nil } \mid \text { ask }: \text { tell } \rightarrow \text { Agent } \mid \text { Agent }+ \text { Agent }
\end{aligned}
$$

where $\leq a n d=$ are the one-way unification and the unification primitives, the $s_{i}^{\prime} s$ and the $t_{i}^{\prime} s$ are Herbrand terms, + is the disjunction and $\rightarrow$ is the commit operator.

$A$ reactive tree is a tree $T$ according to the previous definition, such that each path in $T$ $p\left(X_{1}, \ldots, X_{n}\right):-a s k_{1}:$ tell $_{1} \rightarrow \ldots \rightarrow a s k_{m}:$ tell $_{m}$

satisfies the following condition $(i=1, \ldots, m)$

- if $a s k_{i}=\left\{s_{1} \leq t_{1}, \ldots, s_{n} \leq t_{n}\right\}$ then

$\operatorname{Var}\left(s_{1}, \ldots, s_{n}\right) \cap \operatorname{Var}\left(t_{1}, \ldots, t_{n}\right)=\emptyset$ and

$\operatorname{Var}\left(t_{1}, \ldots, t_{n}\right) \cap \operatorname{Var}\left(H:-a s k_{1}: \operatorname{tell}_{1} \rightarrow a s k_{2}: \operatorname{tell}_{2} \rightarrow a s k_{i-1}: \operatorname{tell}_{i-1}\right)=\emptyset$. 
A path of the form $p\left(X_{1}, \ldots, X_{n}\right):-a s k_{1}:$ tell $_{1} \rightarrow \ldots \rightarrow a s k_{m}:$ tell $l_{m}$ in a reactive tree is called reactive sequence.

The previous condition states that variables which can be instantiated in an ask constraint (local variables) are different from those which cannot (variables coming from the external environment) and reflects the assumptions of the concurrent constraint languages [24,25]. Consider, for example, the following reactive tree (which does not satisfy the above condition)

$$
p(X):-Z \leq X:\{\} \text {. }
$$

The ask constraint $Z \leq X$ specifies that $X$ has to be a variable. This use of one-way unification would raise semantic problems similar to those of the metapredicate $\operatorname{var}(X)$ (for example, in both cases, non-monotonicity arises).

\subsection{The semantics of reactive trees}

A reactive tree specifies all the possible computations for the agent in the root. The basic step of the computation is the ask:tell evaluation performed w.r.t. a global state $\sigma$ (the set of already computed constraints) as specified below.

Definition 3.2 Let $E=\left\{s_{1}=t_{1}, \ldots, s_{n}=t\right\}$ be a set of equations. Then imgu( $\left.E\right)$ is the set of the idempotent most general unifiers of the two terms $\left(s_{1}, \ldots, s_{n}\right)$ and $\left(t_{1}, \ldots, t_{n}\right)$. Moreover let $a s k=\left\{s_{1}^{\prime} \leq t_{1}^{\prime}, \ldots, s_{m}^{\prime} \leq t_{m}^{\prime}\right\}$. Then we define $\Sigma(a s k)=\left\{s_{1}^{\prime}=t_{1}^{\prime}, \ldots, s_{m}^{\prime}=t_{m}^{\prime}\right\}$.

Definition 3.3 (ask : tell evaluation) Let ask $=\left\{s_{1} \leq t_{1}, \ldots, s_{n} \leq t_{n}\right\}$ and tell $=\left\{r_{1}=\right.$ $\left.m_{1}, \ldots, r_{n}=m_{n}\right\}$. Let $\sigma$ be a state (i.e. a consistent set of equations) and $\theta \in$ imgu( $\sigma$ ). Then the evaluation of ask : tell w.r.t. the state $\sigma$ is defined as the result of the following function $\mathcal{E}(a s k: t e l l, \sigma)$

$\mathcal{E}(a s k: t e l l, \sigma)= \begin{cases}\sigma \cup \text { tell } \cup \Sigma(a s k) & \text { if } \exists \theta^{\prime} \in \text { imgu }\left(\left\{\left(s_{1}, \ldots, s_{n}\right) \theta=\left(t_{1}, \ldots, t_{n}\right) \theta\right\}\right) \text { such } \\ & \text { that } \theta^{\prime} \text { does not bind variables in }\left(s_{1}, \ldots s_{n}\right) \theta \text {, and } \\ & \text { imgu }\left(\left\{\left(s_{1}, \ldots, s_{n}\right) \theta=\left(t_{1}, \ldots, t_{n}\right) \theta\right\} \cup \text { tell } \theta\right) \neq \emptyset \\ \text { fail } & \text { if imgu }\left(\left\{\left(s_{1}, \ldots, s_{n}\right) \theta=\left(t_{1}, \ldots, t_{n}\right) \theta\right\} \cup \text { tell } \theta\right)=\emptyset . \\ \text { suspend } & \text { otherwise }\end{cases}$

The semantics of a reactive sequence $s=p\left(X_{1}, \ldots, X_{m}\right):-a s k_{1}:$ tell $_{1} \rightarrow \ldots \rightarrow a s k_{n}: t e l l_{n}$ is the result (answer) of the sequential (from 1 to $n$ ) evaluation of the $a s k_{i}:$ tell $_{i}$ constraints. The evaluation starts with an initial state $\sigma_{i n}$ which represents the external environment. The current state $\sigma_{i}$ is updated by the successful evaluation of an $a s k_{i+1}:$ tell $_{i+1}$ as previously specified. An answer consists of the final state $\sigma_{n}$, restricted to the variables of the root, together with a termination mode (success, fail or deadlock). The formal definition of this semantics is the following.

Definition 3.4 Given an initial store $\sigma_{i n}=\left\{X_{1}=t_{1}, \ldots, X_{m}=t_{m}\right\}$, the answer $\left\langle\Phi, \sigma_{f}\right\rangle$ of the evaluation in $\sigma_{i n}$ of a reactive sequence

$$
p\left(X_{1}, \ldots, X_{m}\right):-a s k_{1}: \operatorname{tell}_{1} \rightarrow \ldots \rightarrow a s k_{n}: \operatorname{tell}_{n}
$$

is defined as follows. Let $\sigma_{0}=\sigma_{\text {in }}$ and let $\sigma_{\mid X}$ denote the state $\sigma$ restricted to the variables in $\left\{X_{1}, \ldots, X_{m}\right\}$. Then

- $\left\langle\Phi, \sigma_{f}\right\rangle=\left\langle\right.$ success, $\left.\sigma_{f}\right\rangle$ if for $i=1, \ldots m, \mathcal{E}\left(a s k_{i}:\right.$ tell $\left._{i}, \sigma_{i-1}\right)=\sigma_{i}$ and $\sigma_{f}=\sigma_{\pi_{1 X}}$, 
- $\left\langle\Phi, \sigma_{f}\right\rangle=\left\langle\right.$ fail,$\left.\sigma_{f}\right\rangle$ if for $i=1, \ldots k, k<n, \mathcal{E}\left(a s k_{i}:\right.$ tell $\left._{i}, \sigma_{i-1}\right)=\sigma_{i}, \mathcal{E}\left(a s k_{k+1}:\right.$ tell $\left._{k+1}, \sigma_{k}\right)=$ fail and $\sigma_{f}=\sigma_{k_{\mid} X}$

- $\left\langle\Phi, \sigma_{f}\right\rangle=\left\langle\right.$ deadlock, $\left.\sigma_{f}\right\rangle$ if for $i=1, \ldots k, k<n, \mathcal{E}\left(a s k_{i}:\right.$ tell $\left._{i}, \sigma_{i-1}\right)=\sigma_{i}, \mathcal{E}\left(a s k_{k+1}\right.$ : tell $\left.l_{k+1}, \sigma_{k}\right)=$ suspend and $\sigma_{f}=\sigma_{k_{\mid X}}$.

The semantics of a reactive tree $T$ is the set of all the possible answers obtained by evaluating the $a s k$ : tell constraints labeling the edges of a path in the tree, starting from the root with a given initial state $\sigma$. The path is obtained by performing a commit at each choice point, i.e. by nondeterministically selecting at each node an edge among those whose evaluation succeeds. As before, the current state is updated by the successful evaluation of an ask and tell element. A successful answer of $T$ in $\sigma$ is then the succesful result of the evaluation in $\sigma$ of any reactive sequence of $T$. It is worth noting that a reactive sequence is a path starting in the root of the tree and ending in a node, possibly different from a leaf of the tree. Therefore, the semantics we consider include also the results of partial successful computations. Finite failure and deadlock answers are defined by considering the structure of the node $N$ reached by a succesful reactive sequence $s$. Namely, if $\sigma_{n}$ is the state obtained by the successful evaluation of $s$, we have a failure answer if the evaluation of all the (ask : tell labels of the) edges of $N$ fail. The answer is a deadlock if all the edges of $N$ are suspended or failed, and there exists at least one suspended edge. Let us give the formal definition of the semantics of a reactive tree in terms of its answers. In the following $\left(\sum_{j \in J} a s k_{n+1}^{j}: t e l l_{n+1}^{j}\right)$ stands for a node whose outcoming edges are labelled by $a s k_{n+1}^{j}: t e l l_{n+1}^{j}$ for $\supset \in J$.

Definition 3.5 Let $\sigma_{0}=\left\{X_{1}=t_{1}, \ldots, X_{m}=t_{m}\right\}$ be an initial state, let $T$ be a reactive tree with root $p\left(X_{1}, \ldots, X_{m}\right)$ and let $\sigma_{\mid X}$ denote the state $\sigma$ restricted to the variables in $\left\{X_{1}, \ldots, X_{m}\right\}$. Then $\left\langle\Phi, \sigma_{f}\right\rangle$ is an answer for $T$ in the initial state $\sigma_{i n}$ if one of the following cases holds

- $\left\langle\Phi, \sigma_{f}\right\rangle=\left\langle\right.$ success, $\left.\sigma_{f}\right\rangle$, where $\left\langle\right.$ success, $\left.\sigma_{f}\right\rangle$ is a (success) answer for any reactive sequence in $T$,

- $\left\langle\Phi, \sigma_{f}\right\rangle=\left\langle\right.$ fail,$\left.\sigma_{f}\right\rangle$ if there exists a subtree

$$
p\left(X_{1}, \ldots, X_{m}\right):-a s k_{1}: \operatorname{tell}_{1} \rightarrow \ldots \rightarrow a s k_{n}: \operatorname{tell}_{n} \rightarrow\left(\sum_{j \in J} a s k_{n+1}^{j}: \operatorname{tell}_{n+1}^{j}\right)
$$

of $T$, such that for $i=1, \ldots n, \mathcal{E}\left(a s k_{i}:\right.$ tell $\left._{i}, \sigma_{i-1}\right)=\sigma_{i}$, for $j \in J, \mathcal{E}\left(a s k_{n+1}^{j}:\right.$ tell $\left._{n+1}^{j}, \sigma_{n}\right)=$ fail and $\sigma_{f}=\sigma_{n_{\mid X}}$,

- $\left\langle\Phi, \sigma_{f}\right\rangle=\left\langle\right.$ suspend, $\left.\sigma_{f}\right\rangle$ if there exists a subtree

$$
p\left(X_{1}, \ldots, X_{m}\right):-a s k_{1}: \operatorname{tell}_{1} \rightarrow \ldots \rightarrow a s k_{n}: \operatorname{tell}_{n} \rightarrow\left(\sum_{j \in J} a s k_{n+1}^{j}: \operatorname{tell}_{n+1}^{j}\right)
$$

of $T$, such that for $i=1, \ldots n, \mathcal{E}\left(a s k_{i}:\right.$ tell $\left._{i}, \sigma_{i-1}\right)=\sigma_{i}$, for $j \in J, \mathcal{E}\left(a s k_{n+1}^{j}: t e l l_{n+1}^{j}, \sigma_{n}\right) \in$ $\{$ fail, suspend $\}, \exists \bar{j} \in J$ such that $\mathcal{E}\left(a s k_{n+1}^{\tilde{j}}:\right.$ tell $\left.{ }_{n+1}^{\bar{j}}, \sigma_{n}\right)=$ suspend and $\sigma_{f}=\sigma_{n_{\mid X}}$.

\section{A solved form for ask and tell constraints}

A solved form for equations and an algorithm for computing it was defined in [16]. A solved form for one-way unifications was introduced in [8]. In this section we will give the definitions of solved form for ask:tell constraints together with an algorithm to compute it which is proved to be correct. The semantics of an ask constraint $\left\{s_{1} \leq t_{1}, \ldots, s_{n} \leq t_{n}\right\}$ is obtained, according to definition 3.3, by evaluating $s \leq t$ where $s=\left(s_{1}, \ldots, s_{n}\right)$ and $t=\left(t_{1}, \ldots, t_{n}\right)$. Moreover we are concerned with ask 
constraints such that $\operatorname{Var}\left(s_{1}, \ldots, s_{n}\right) \cap \operatorname{Var}\left(t_{1}, \ldots, t_{n}\right)=($ definition 3.1 of reactive tree). Hence in the following we will consider ask constraints of the form $s \leq t$ where $s$ and $t$ do not share variables. The notion of ask and tell evaluation (definition 3.4) is given w.r.t. a state $E$. Therefore in the following we will consider the notion of ask : tell solved form w.r.t. a set of variables V, whose bindings represent the state in which the ask $:$ tell constraint is evaluated. (Equivalently we can consider elements of the form $p\left(X_{1}, \ldots, X_{n}\right):-a s k$ : tell where $\left.V=\left\{X_{1}, \ldots, X_{n}\right\}\right)$.

Definition 4.1 (solved form of constraints) An ask : tell constraint is in solved form w.r.t. the set of variables $V$ iff the following conditions hold:

i) $a s k=\left\{X_{1} \leq t_{1}, \ldots, X_{n} \leq t_{n}\right\}$ or ask $=\{$ suspend $\}$.

tell $=\left\{Y_{1}=s_{1}, \ldots, Y_{m}=s_{m}\right\}$,

ii) the $X_{i}^{\prime} s, Y_{i}^{\prime} s$ are distinct variables,

iii) $\left\{X_{1}, \ldots, X_{n}\right\} \cap \operatorname{Var}\left(t_{1}, \ldots t_{n}\right)=\emptyset$ and $\left\{Y_{1}, \ldots, Y_{m}\right\} \cap \operatorname{Var}\left(s_{1}, \ldots s_{m}\right)=\emptyset$,

iv) $\left\{X_{1}, \ldots, X_{n}\right\} \cap \operatorname{Var}\left(s_{1}, \ldots s_{m}\right)=\emptyset$ and $\operatorname{Var}\left(t_{1}, \ldots t_{n}\right) \cap \operatorname{Var}\left(s_{1}, \ldots s_{m}\right)=\emptyset$,

iv) $\left\{X_{1}, \ldots, X_{n}\right\} \subseteq V,\left\{Y_{1}, \ldots, Y_{m}\right\} \subseteq\left(V \cup \operatorname{Var}\left(t_{1}, \ldots t_{n}\right)\right)$,

v) the set of equations $\left\{X_{1}=t_{1}, \ldots, X_{n}=t_{n}, Y_{1}=s_{1}, \ldots, Y_{m}=s_{m}\right\}$ is unifiable.

The element suspend stands for an always suspending constraint. Let us show a simple example of the solved form of an ask and tell constraint.

\section{Example 4.2}

$$
p(X, Y):-\{f(X) \leq f(g(Z))\}:\{X=g(a), X=Y\}
$$

The solved form of the previous ask and tell constraint (w.r.t the variables $\{X, Y\}$ ) is

$$
p(X, Y):-\{X \leq g(Z)\}:\{Z=a, Y=g(a)\}
$$

In the following we describe how to compute the solved form of an ask and tell constraint. As pointed out in [22], the primitive $\leq$ (one-way unification) is semantically very close to the partial ordering of subsumption ( $\supseteq$ ) among terms. In particular if we consider idempotent substitutions, $s \leq t$ succeeds iff $t \supseteq s$. We will then use some notions related to subsumption which have been introduced in [22].

Definition 4.3 Let $\theta=\left\{X_{1} / t_{1}, \ldots, X_{n} / t_{n}\right\}$ be a susbstitution. Then $\operatorname{Dom}(\theta)=\left\{X_{1}, \ldots, X_{n}\right\}$ and $\operatorname{Cod}(\theta)=\operatorname{Var}\left(t_{1}, \ldots, t_{n}\right)$. If $E=\left\{s_{1}=t_{1}, \ldots, s_{n}=t_{n}\right\}$ (or $E=\left\{s_{1} \leq t_{1}, \ldots, s_{n} \leq t_{n}\right\}$ ), $\operatorname{Dom}(E)=\operatorname{Var}\left(s_{1}, \ldots, s_{n}\right)$ and $\operatorname{Cod}(E)=\operatorname{Var}\left(t_{1}, \ldots, t_{n}\right)$. If $t$ is a term, $\theta_{\mid t}(\theta$ restricted to $t)$ is defined as $\theta_{\mid t}=\{X / m \mid X / m \in \theta$ and $X \in \operatorname{Var}(t)\}$. Finally $\rho: \operatorname{Var} \rightarrow \operatorname{Var}$ is a renaming iff there exists $\rho^{-1}$ such that $\rho \rho^{-1}=\rho^{-1} \rho=\epsilon$ where $\epsilon$ is the empty substitution (i.e. $\rho$ is a bijective function).

Definition 4.4 [22] Let $s$ and $t$ be first order terms. $t$ subsumes $s$, ( $t \supseteq s)$ iff there exists an idempotent substitution $\theta$ such that $t \theta=s$.

Definition 4.5 [22] Let $s$ and $t$ be first order terms. An orderer of $t$ over $s$ is an idempotent substitution $\theta$ such that $t \theta \supseteq s \theta . \theta$ is a most general orderer (mgo) of $t$ over $s$ iff for any other orderer $\sigma$ there exists a substitution $\mu$ such that $\theta \mu=\sigma$.

The following definition of impartial substitution is modified from that originally given in [22]. 
Definition 4.6 Let $S$ be a set of first order terms. An impartial substitution for $S$ is a substitution $\theta$ such that $\operatorname{Cod}\left(\theta_{\mid S}\right) \cap \operatorname{Var}(S)=\emptyset$ and $\theta_{\mid S}$ is idempotent. An impartial unifier for $s$ and $t$ is a unifier for $s$ and $t$ which is impartial for $\{s, t\}$.

The most general impartial unifier is defined as usual, namely $\theta$ is the most general impartial unifier for two terms $s$ and $t$ iff for each impartial unifier $\gamma$ of $s$ and $t$ there exists a substitution $\sigma$ such that $\gamma=\theta \sigma$. The following lemma shows that two terms $s$ and $t$ are unifiable iff they have a most general impartial unifier (mgiu).

Lemma 4.7 Let $s$ and $t$ be terms. Then there exists an mgiu iff $s$ and $t$ are unifiable. Moreover $\beta$ is 'an mgiu for $t$ and $s$ iff $\beta=\theta \rho$, where $\theta=\left\{X_{1} / u_{1}, \ldots, X_{n} / u_{n}\right\}$ is an idempotent mgu for $\{s, t\}$ and $\rho$ is a renaming such that $\operatorname{Cod}\left(\rho_{\mid U}\right) \cap \operatorname{Var}(\{s, t\})=\emptyset$, where $U=\left\{u_{1}, \ldots, u_{n}\right\}$.

\section{Proof}

Let $\theta$ be an idempotent mgu for $s$ and $t$ and let $\rho$ be a renaming as defined in the hypothesis of the lemma. Then $\theta \rho$ is an impartial unifier for $s$ and $t$. Moreover let $\gamma$ be an impartial unifier for $s$ and $t$. Since $\theta$ is an mgu for $\mathrm{s}$ and $\mathrm{t}$ we have $\gamma=\theta \alpha$ and $\gamma=\theta \rho \rho^{-1} \alpha$. Therefore $\theta \rho$ is the most general impartial unifier. Conversely, let $\beta$ be an mgiu for $s$ and $t$. Then $s$ and $t$ are unifiable and there exists an idempotent mgu $\theta$. Since $\theta$ is an mgu, $\beta=\theta \sigma$ for some $\sigma$. Since $\theta \rho$ (where $\rho$ is defined as before) is an impartial unifier and $\beta$ is the most general, $\sigma$ is a renaming. Since $\beta$ is impartial $\operatorname{Cod}\left(\sigma_{\mid U}\right) \cap \operatorname{Var}(\{s, t\})=\emptyset$. If $s$ and $t$ are not unifiable obviously there exists no impartial unifier for $s$ and $t$. If there exists no mgiu for $s$ and $t$ then (by the previous part of the proof) there exists no unifier for $s$ and $t$. This completes the proof.

Note that the original definition of impartial substitution was given in [22] as follows.

Definition 4.8 [22] An impartial substitution for $S$ is an idempotent substitution $\theta=\left\{X_{1} / u_{1}, \ldots, X_{n} / u_{n}\right\}$ where no $u_{i}$ contains variables of any term in $S$. An impartial unifier for two terms $s$ and $t$, is a unifier of $s$ and $t$ which is impartial for $\{s, t\}$.

In [22] it is claimed that the most general impartial unifier of $s, t$ can be obtained by considering an idempotent mgu of $s, t$ which is impartial for $\{s, t\}$. The following lemma shows that, according to the previous definition, there exists no unique (up to variable renaming) most general impartial unifier of two terms $s$ and $t$.

Lemma 4.9 In general there exists no (most general) impartial unifier (according to definition 4.8 of impartial unifier) $\theta$ for two terms $s$ and $t$, such that for every impartial unifier $\gamma$ of $s$ and $t, \gamma=\theta \sigma$ for some $\sigma$.

\section{Proof}

Let us consider two terms $X$ and $Y$. Let $\gamma=\{X / M, Y / M\}, M \neq X, M \neq Y$, and let us show that no impartial substitution (according to definition 4.8) $\theta \neq \gamma$ for $X$ and $Y$ is more general than $\gamma$. Let us suppose that $\gamma=\theta \sigma$ where $\theta \neq \gamma$ is impartial for $X$ and $Y$. Since any impartial substitution $\beta$ for $\{X, Y\}$ is such that $\beta \supseteq \gamma=\{X / Z, Y / Z\}$ for some $Z$ with $Z \neq X, Z \neq Y$, let us suppose, without loss of generality, that $\theta \supseteq \theta^{\prime}=\{X / Z, Y / Z\}$ where $Z \notin \operatorname{Dom}(\gamma)$. Since $\theta$ is impartial, $Z / Y \notin \theta$. Then $\sigma \supseteq\{Z / M\}$ and hence one of the following cases hold

- if $Z / m \in \theta$, where $m$ is not a variable, then $Z / m \sigma \in \theta \sigma$,

- if $Z \notin \operatorname{Dom}(\theta)$ then $\{Z / M\} \in \theta \sigma$,

- if $Z / W \in \theta, W \neq Y$ then either $Z / W \in \theta \sigma$ or $W / Z \in \theta \sigma$ (and we can repeat the same argument for $W$ ). 
Then in all the three cases, since $Z \notin \operatorname{Dom}(\gamma), \theta \sigma \neq \gamma^{\prime}$ contradicting the original hypothesis. Moreover $\gamma^{\prime}$ is not the most general impartial unifier, since the previous considerations show that, for example, if we consider $\theta^{\prime}=\{X / Z, Y / Z\}$ as impartial unifier of $X$ and $Y, \theta^{\prime} \neq \gamma \sigma$ for any $\sigma$.

Note that the substitution $\rho=\{Z / M\}$ is not a renaming (there exists no $\rho^{-1}$ ), while $\{Z / M, M / Z\}$ is a renaming. This obvious consideration allows to define the most general impartial unifier as shown in lemma 4.7. Then if we consider the terms $X$ and $Y$ as in the previous proof, their most general impartial unifer (up to renaming) is $\theta=\{X / M, Y / M, M / Y\}$ and, for example, we have $\alpha=\{X / Z, Y / Z\}=\theta \sigma$ where $\sigma=\{M / Z, Y / M\}$. Moreover note that if $\rho=\{M / Z, Z / M\}$ is a renaming, then $\theta \rho=\{X / Z, Y / Z, M / Y, Z / M\}$ is still a most general impartial unifier (for example $\alpha=\theta \rho \gamma$ where $\gamma=\{Y / M, M / Z\}$.

Definition 4.10 [22] Let $\theta$ be a unifier for $s$ and $t$. Then $\theta_{[s, t]}$ is defined as $\theta_{[s, t]}=\{X / u \mid X / u \in \theta$ and $X$ is a variable that appears in $t\}$.

The following theorem is a modified (weaker) version of a theorem in [22] and shows how to obtain an orderer for $t$ over $s$ computing the most general impartial unifier (mgiu) for $t$ and $s$. The proof is the same as in [22].

Theorem 4.11. [22] Let $s$ and $t$ be two terms. Then if $\theta$ is a most general impartial unifier for $s$ and $t, \theta_{[s, t]}$ is an orderer of $s$ over $t$ (i.e. $\left.s \theta_{[s, t]} \supseteq t \theta_{[s, t]}\right)$.

Note that the stronger original version of [22] which gives $\theta_{[s, t]}$ as the most general orderer does not hold. As a countexample, consider the terms $s=f(X)$ and $t=Y$. Then $\theta_{[s, t]}=\{Y / f(M)\}$ is an orderer of s over $\mathrm{t}$, but not the most general, since $\beta=\{Y / f(Z)\}$ is an orderer of $\mathrm{s}$ over $\mathrm{t}$ and $\beta \neq \theta_{[s, t]} \sigma$ for any $\sigma$. The most general orderer is $\{Y / f(M) M / X\}$ which is not idempotent.

Corollary 4.12 Let $s$ and $t$ be two terms which do not share variables and let $\theta$ be a most general impartial unifier for $s$ and $t$. Then $s \supseteq t \theta_{[s, t]}$. Moreover, if $t \supseteq s$ then $t \theta_{[s, t]}=s \theta_{[t, s]}$ and $\theta_{[t, s]}$ is only a variable renaming.

\section{Proof}

The first part is a straightforward consequence of theorem 4.11, since $s \theta_{[s, t]}=s$. By definition $\theta$ is an mgiu for $\{s, t\}$ and since $s$ and $t$ do not share variables $t \theta_{[s, t]}=s \theta_{[t, s]}$. Moreover, since by hypothesis $t \supseteq s$, there exists, by definition, an idempotent unifier $\beta$ for $s$ and $t$ such that $t \beta=s$. Hence, if $\rho$ is a renaming which introduces variables not in $\{t, s\}$, then $\beta \rho$ is by lemma 4.7 an impartial unifier for $s$ and $t$. Since $\beta$ does not instantiate variables in $s, s \beta \rho=s \rho$. Since $\theta$ is the most general impartial unifier for $\{s, t\}, s \beta \rho=s \rho=s \theta \gamma=s \theta_{[t, s]} \gamma$. Therefore $\theta_{[t, s]}$ is a variable renaming.

\subsection{The ask:tell solved form algorithm}

In this subsection we will give the algorithm which computes the solved form of an ask : tell constraint and we will show that it is correct, i.e. it preserves the semantics of the constraint.

Definition 4.13 Given two terms $t=f\left(t_{1}, \ldots, t_{n}\right)$ and $s=f\left(s_{1}, \ldots, s_{n}\right)$, a subterm $l_{t}$ of $t$ corresponds in $s$ to a subterm $l_{s}$ (of $s$ ) iff either

- $l_{t}=t_{i}$ and $l_{s}=s_{i}$ or

- $l_{t}$ is a subterm of $t_{i}, l_{s}$ is a subterm of $s_{i}$ and $l_{i}$ corresponds in $s_{i}$ to $l_{s}$. 
Let us consider an ask : tell constraint $\{s \leq t\}:$ tell where tell is a set of (standard) equations and $\operatorname{Var}(s) \cap \operatorname{Var}(t)=\emptyset$. If $s$ and $t$ are not unifiable, the primitive $s \leq t$ always fails and the solved form of the whole constraint results in the value fail (which stands for an always failing constraint). Otherwise the solved form of $s \leq t$ can be computed by noting that if $\theta=\theta_{[s, t]} \cup \theta_{[i, s]}$ is an mgiu for $s$ and $t$, then $s \supseteq t \theta_{[s, t]}$ (corollary 4.12). Therefore $s \leq t \theta_{[s, t]}$ can be simplified to a set of atoms in solved form $\left\{X_{1} \leq t_{1}, \ldots, X_{n} \leq t_{n}\right\}$ where $X_{i} \in s$ and $t_{i}$ is the subterm of $t$ corresponding to $X_{i}$ (definition 4.13 ), $i=1, \ldots n$. The solved form of the tell part, is performed by applying the well known solved form algorithm $[21,16]$ to the set of equations $E$ modified as above specified. Let us now give the algorithm.

begin

The solved form $a s k_{n}: t e l l_{n}$ of the constraint $s \leq t: t e l l$, w.r.t. the set of variables $V$ is computed as follows:

i) If $s$ and $t$ are not unifiable, the primitive $s \leq t$ always fails. Therefore the solved form of the whole constraint results in the value fail.

ii) If $s$ and $t$ are unifiable, since $s$ and $t$ do not share variables, by corollary 4.12 , if $\theta=\theta_{[s, t]} \cup \theta_{[t, s]}$ is an mgiu for $s$ and $t$, then $\theta_{[s, t]}$ is an orderer of $s$ over $t$, i.e. $s \supseteq t \theta_{[s, t]}$. Then we define $I_{1}=\left\{Y \leq m \mid Y \in \operatorname{Var}(s)\right.$ and $Y$ corresponds in $t \theta_{[s, t]}$ to the term $\left.m\right\}$

$A=\left\{Y \leq M \mid Y \leq M \in I_{1}, Y \in V, M\right.$ is a variable, there exists no $X \leq m \in I_{1}$ such that $X \in V, X \neq Y$ and the variable $M$ appears in $m\}$

$B=\left\{Y \leq M \mid Y \leq M \in I_{1}, M\right.$ is a variable, $\left.Y \notin V\right\}$.

$C=\left\{Y \leq m \in I_{1} \mid m\right.$ is not a variable and $\left.Y \notin V\right\}$.

$D=\left\{Y \leq X \mid Y \notin V, \exists Z \leq t \in I_{1}\right.$ with $Z \neq Y$ and $X$ appears in $\left.t\right\}$.

Let $I_{2}=A \cup B$ and $I_{3}=C \cup D$. Let us distinguish two cases

1. (success) if $I_{3}=0$ then the solved form of ask constraint is ask $k_{n}=I_{1} \backslash I_{2}$.

2. (suspension) Let $I_{3} \neq \emptyset$. Then the solved form of the ask constraint is $a s k_{n}=\{s u s p e n d\}$.

The bindings computed by $\theta_{[s, t]}$ have to be passed to the set of equations in the tell part. Then we define:

$$
t_{e l l}=\operatorname{tell} \theta_{[s, t]} .
$$

Moreover, in order to obtain a solved form sequence, also the bindings defined by $I_{1}$ have to be passed. Therefore we define

$$
\begin{aligned}
& \text { tell }_{2}=\text { tell }_{1} \alpha \text { where } \\
& \alpha=\left\{X / t \mid X \leq t \in I_{1} \backslash A\right\} \cup\{M / Y \mid Y \leq M \in A\} .
\end{aligned}
$$

iii) The solved form of the tell part, is performed by applying the well known solved form algorithm $[21,16]$ to the set of equations of the tell modified as above specified. Let Solved-form $(X)$ be the result of the application of the solved-form algorithm to the set of equations $X$. Then

1. If tell $_{2}$ is not unifiable then the solved form of the whole constraint is the value fail.

2. If tell $_{2}$ is unifiable then $\operatorname{tell}_{n}=\operatorname{Solved-form}\left(\operatorname{tell}_{2}\right) \backslash\{F \cup G\}$ where:

$$
\left.\begin{array}{ll}
F=\{X=t \mid & \left.X=t \in \text { Solved-form }\left(\operatorname{tell}_{2}\right) \text { and } X \notin V \cup \operatorname{Var}\left(a s k_{n}\right)\right\} \\
G=\{X=Y \mid & X=Y \in \operatorname{Solved-form}\left(\operatorname{tell}_{2}\right), X \in V \\
& Y \notin V \cup \operatorname{Var}\left(a s k_{n}\right), X \in V \cup \operatorname{Var}\left(a s k_{n}\right)
\end{array}\right\} .
$$

end. 


\subsection{Notes about the algorithm}

1. Since $s \supseteq t \theta_{[s, t]}, s$ differs from $t \theta_{[s, t]}$ only because for a subterm $s_{1}=f(\ldots, X, \ldots)$ of $s$, there exists a corresponding subterm (as defined in definition 4.13) $t_{1}=f\left(\ldots, t_{h}, \ldots\right)$ in $t \theta_{[s, t]}$ where $t_{h}$ is a term. So the set $I_{1}$ at step $\left.i i\right)$ is well defined.

2. The elements of the set $I_{2}$ defined at step $\left.i i\right)$, can be deleted since they do not define constraints. Moreover note that, because of the definitions of $I_{2}$, we do not further reduce ask constraints of the form $p(X, Y):-\{X \leq f(Z), Y \leq Z\}:\{\}$.

In fact we cannot reduce the constraint to $\{X \leq f(Y)\}$ since the resulting sequence $p(X, Y):-\{X \leq f(Y)\}:\{\}$ would not be legal.

3. Cases of suspension considered at step ii) are those of the form:

s) $p(X):-\{Y \leq f(a)\}:$ tell

s2) $p(X):-\{Z \leq K, X \leq f(K)\}:$ tell

$\left.s_{3}\right) p(X):-\{Z \leq K, M \leq K\}:$ tell

The ask constraints in $s_{1}, s_{2}$ and $s_{3}$ are always indefinitely suspended, since $Y, Z$ and $M$ cannot be instantiated. Therefore the solved form of an ask constraint containg these forms of atoms, will be suspend. The semantics of the whole ask constraint will result in a suspension or in a failure depending on the other constraints, which therefore have to appear in the solved form of the ask constraint.

4. A substitution obtained by the computation of the solved form of the ask constraint is applied to the tell part. Indeed the bindings in $\theta_{[s, t]}$ and $\alpha$ have to be applied in order to preserve the semantics since some of them they do not appear in the solved form of the ask constraint. The bindings $\left\{X_{1} / t_{1}, \ldots, X_{n} / t_{n}\right\}$ which are determined by the solved version of the ask constraint are also applied to the tell part to prevent further occurrences of the $X_{i}^{\prime} s$, in order to obtain ask : tell constraint in solved form.

5. The atoms in the sets $F$ and $G$ defined at step $i i i)$ are deleted since they define bindings for variables which are not in the previous part of the sequence.

\subsection{The equivalence of an ask:tell constraint and its solved form}

The following lemma is an obvious consequence of the definition of the previous algorithm.

Lemma 4.14 An ask : tell constraint modified as specified by the previous algorithm is in solved form (according to definition 4.1).

Let us now show the equivalence between an ask $:$ tell constraint and its solved form. We first need some lemmata.

Lemma 4.15 Let $s$ and $t$ be first order terms with $\operatorname{Var}(t) \cap \operatorname{Var}(s)=\emptyset$. Let $\theta$ be an idempotent $m g u$ of $s$ and $t$ and let $\alpha$ be a substitution such that $\operatorname{Dom}(\alpha) \subseteq \operatorname{Var}(s)$. Then

- if $s \alpha$ and $t$ are unifiable then $\theta_{[s, t]} \sigma$ is a unifier of s $\alpha$ and $t$ for some $\sigma$.

- if there exists an idempotent mgu $\gamma$ of s $\alpha$ and $t$ which does not bind variables in s $\alpha$, then $\gamma=\left(\theta_{[s, t]} \sigma\right)_{\mid t}$ for some $\sigma$. 


\section{Proof}

The first part is a direct consequence of the definition of the unification algorithm. If $X / m \in \theta_{[s, t]}$ then $X /(m \alpha) \in \gamma$. Conversely if $X / m \in \gamma$ either $X / l \in \theta_{[s, t]}$ and $m=l \alpha$, or $X \notin \operatorname{Dom}\left(\theta_{[s, t]}\right)$. If $\sigma^{\prime}$ $=\left\{X / l \in \gamma \mid X \notin \operatorname{Dom}\left(\theta_{[s, t]}\right)\right\}$, then, since $\alpha$ does not bind variables in $t$ and since $t$ and $s$ do not share variables, $\gamma=\left(\theta_{[s, t]} \alpha \sigma^{\prime}\right)_{\mid t}$ and the thesis holds.

Lemma 4.16 Let $s$ and $t$ be first order terms and let $\operatorname{Var}(t) \cap \operatorname{Var}(s)=\emptyset$. If there exists a unifier $\theta$ of $s$ and $t$ which does not bind variables in $s$, then $\theta_{\mid t}$ is idempotent and unique.

\section{Proof}

Obviously the only unifier $\theta_{\mid t}$ for $\mathrm{s}$ and $\mathrm{t}$ which does not bind variables in $s$ is given by $\theta=$ $\left\{X_{1} / t_{1}, \ldots, X_{n} / t_{n}\right\}$, where, for $i=1, \ldots, n, X_{i}$ in $t$ corresponds to $u_{i}$ in $s$ according to definition 4.13. $\theta$ is idempotent since $\operatorname{Var}(t) \cap \operatorname{Var}(s)=\emptyset$.

Lemma 4.17 Let $s$ and $t$ be first order terms and let $\operatorname{Var}(t) \cap \operatorname{Var}(s)=\emptyset$. Let $\theta_{[s, t]}$ be an orderer of $s$ over $t$ (definition 4.10) and let $\alpha$ be a subsitution such that $\operatorname{Dom}(\alpha) \subseteq \operatorname{Var}(s)$ and $\operatorname{Cod}(\alpha) \cap\left(\operatorname{Var}(t) \cup \operatorname{Var}\left(\theta_{[s, t]}\right)\right)=\emptyset$. Then $s \leq t$ is equivalent to $s \leq t \theta_{[s, t]}$, i.e.

- $s \alpha \leq t$ succeeds, fails or suspends iff $s \alpha \leq t \theta_{[s, t]}$ succeeds, fails or suspends,

- $s \alpha \leq t$ succeeds with c.a.s. $\beta$ iff $s \alpha \leq t \theta_{[s, t]}$ succeeds with c.a.s. $\gamma$ such that $t \theta_{[s, t]} \gamma=t \beta$.

\section{Proof}

Let $\theta$ be an mgiu for $\{s, t\}$ and let $\theta_{[s, t]}, \theta_{[t, s]}$ be as defined in definition 4.10. Since $\operatorname{Var}(s) \cap \operatorname{Var}(t)=$ $\emptyset, \theta_{[s, t]}$ binds variables in $t$ only and $\theta_{[t, s]}$ binds variables in $s$ only. By definition of mgiu and by lemma $4.7 \theta=\theta^{\prime} \rho$ where $\theta^{\prime}=\left\{X_{1} / u_{1}, \ldots, X_{n} / u_{n}\right\}$ is an idempotent mgu for $\mathrm{s}$ and $t$ and $\rho$ is a renaming such that $\operatorname{Cod}\left(\rho_{\mid U}\right) \cap \operatorname{Var}(\{s, t\})=\emptyset$, where $U=\left\{u_{1}, \ldots, u_{n}\right\}$, (i.e. $\rho$ renames variables in $\left\{u_{1}, \ldots, u_{n}\right\}$ with fresh ones). Let us consider two cases.

1. If $s \alpha \leq t$ fails, by definiton of $\leq, s \alpha$ and $t$ are not unifiable, hence $s \alpha$ and $t \theta_{[s, t]}$ are not unifiable too and $s \alpha \leq t \theta_{[s, t]}$ fails. Conversely if $s \alpha \leq t \theta_{[s, t]}$ fails let us suppose that $s \alpha$ and $t$ are unifiable. By lemma $4.15 \theta_{[s, t]}^{\prime} \sigma$ is a unifier of $s \alpha$ and $t$. Moreover $\theta_{[s, t]}^{\prime} \sigma=\theta_{[s, t]}^{\prime} \rho \rho^{-1} \sigma=$ $\theta_{[s, t]} \rho^{-1} \sigma$ by definition of $\theta$ (since $\rho$ is a renaming we can define $\rho^{-1}$ ) and $t \theta_{[s, t]}=t \theta_{[s, t]} \theta_{[s, t]}$, because $\theta_{[s, t]}$ is idempotent. Hence we have that

$$
\begin{aligned}
& s \alpha \theta_{[s, t]}^{\prime} \sigma= \\
& s \alpha \theta_{[s, t]} \rho^{-1} \sigma= \\
& t \theta_{[s, t]} \rho^{-1} \sigma= \\
& t \theta_{[s, t]} \theta_{[s, t]} \rho^{-1} \sigma=
\end{aligned}
$$

and $s \alpha, t \theta_{[s, t]}$ would be unifiable, thus contradicting the fact that $s \alpha \leq t \theta_{[s, t]}$ fails.

2. Let us suppose that $s \alpha \leq t$ succeeds with c.a.s. $\beta$, i.e. $\beta$ is an idempotent mgu of $s \alpha$ and $t$ and $\beta$ does not bind variables in $s \alpha$. Therefore $\beta=\left(\theta_{[s, t]}^{\prime} \gamma^{\prime}\right)_{\mid t}$ (lemma 4.15) and obviously $\theta_{[s, t]}^{\prime}$ does not bind variables in $s \alpha$. As before, $\theta_{[s, t]}^{\prime}=\theta_{[s, t]} \rho^{-1}$, hence $\beta=\left(\theta_{[s, t]} \rho^{-1} \gamma^{\prime}\right)_{\mid t}=$ $\theta_{[s, t]} \rho_{\left[\operatorname{Cod}\left(\theta_{[s, s]}\right)\right.}^{-1} \gamma_{\left[\operatorname{Dom}\left(\rho^{-1}\right)\right.}^{\prime}$ since, by definition of $\rho$, all the variables in $t \theta_{[s, t]}$ are bound to terms which contain fresh variables, i.e. there does not exist $Y \in t \theta_{[s, t]}$ with $Y \notin \operatorname{Dom}\left(\rho^{-1}\right)$. Therefore let $\rho_{\mid \operatorname{Cod}\left(\theta_{[s, t]}\right)}^{-1} \gamma_{\mid \text {Dom }\left(\rho^{-1}\right)}^{\prime}=\gamma$. Hence

$$
\begin{array}{ll}
s \alpha & = \\
s \alpha \beta & = \\
s \alpha \theta_{[s, t]} \gamma & = \\
t \beta & = \\
t \theta_{[s, t]} \gamma . &
\end{array}
$$


By definition $\gamma$ does not bind variables in $s \alpha$ and is a unifier of $s \alpha$ and $t \theta_{[s, t]}$ with $t \theta_{[s, t]} \gamma=$ $t \beta$. Moreover, by lemma 4.16 if $\delta$ is any mgu for $s \alpha$ and $t \theta_{[s, t]}$ and $\delta$ does not bind variables in $s \alpha, \delta_{\mid t \theta_{[s, t]}}=\gamma_{\mid t \theta_{[s, t]}}=\gamma$, hence the "if" part holds.

For the "only if" part, let $\gamma^{\prime}$ be an idempotent unifier of $s \alpha$ and $t \theta_{[s, t]}$ which does not bind variables in $s \alpha$. Let us consider the substituion $\delta$ defined as $\delta=\theta_{[s, t]} \gamma^{\prime}$. Since $s \alpha=t \delta_{\mid t}$ and $\delta_{\mid t}$ does not bind variables in $s \alpha$, by lemma 4.16 if $s \alpha \leq t$ succeeds with c.a.s. $\beta, \beta_{\mid t}=\delta_{\mid t}$ and obviously $t \beta=t \theta_{[s, t]} \gamma^{\prime}$.

We have thus shown that $s \leq t$ is equivalent to $s \leq t \theta_{[s, t]}$ for successes and failures. Therefore the equivalence holds also for suspension.

The following theorem states the equivalence between an ask: tell constraint and its solved form.

Definition 4.18 Let $c_{1}=H:-a s k_{1}:$ tell $_{1}$ and $c_{2}=H:-a s k_{2}:$ tell $l_{2}$. Then $c_{1}$ and $c_{2}$ are equivalent iff, given an initial state $\sigma_{\text {in }}$, i.e. a set of bindings for the variables in $H$, the following holds

- the evaluation of ask : tell in $\sigma_{\text {in }}$ yields the answer $\langle\Phi, \sigma\rangle(\Phi \in\{$ success, fail, deadlock $\})$ (definition 3.4), iff the evaluation of ask $k_{n}$ : tell $_{n}$ in $\sigma_{\text {in }}$ yields the answer $\left\langle\Phi^{\prime}, \sigma^{\prime}\right\rangle$ with $\Phi=\Phi^{\prime}$ and $\theta=\theta^{\prime} \rho$, where $\theta$ is an idempotent mgu of $\sigma, \theta^{\prime}$ is an idempotent mgu of $\sigma^{\prime}$ and $\rho$ is a renaming.

Theorem 4.19 Let $c_{s o l}=H:-a s k_{n}: t e l l_{n}$ be the solved form of $c=H:-a s k$ : tell (w.r.t. $\operatorname{Var}(H))$ as obtained by the previously specified algorithm, where ask $=\{s \leq t\}$ and $\operatorname{Var}(s) \cap$ $\operatorname{Var}(t)=\emptyset$. Then $c$ and $c_{\text {sol }}$ are equivalent (definition 4.18).

\section{Proof}

If $H=p\left(X_{1}, \ldots X_{k}\right)$ let $\alpha$ be the substitution $\left\{X_{1} / t_{1} \ldots X_{k} / t_{k}\right\}$ which represents the initial state $E_{\text {in }}$, with $\operatorname{Var}\left(t_{1}, \ldots, t_{h}\right) \cap(\operatorname{Var}(s) \cup \operatorname{Var}(t))=\emptyset$. Then the thesis follows from the following arguments

1. by lemma 4.17 the evaluation of $s \alpha \leq t$ is equivalent to that of $s \alpha \leq t \theta_{[s, t]}$ w.r.t. $\operatorname{Var}(t)$ (where $\theta_{[s, t]}$ is defined as in definition 4.10). Moreover, according to the semantics, $\left\{f\left(X_{1} \ldots, X_{n}\right) \leq\right.$ $\left.f\left(t_{1} \ldots, t_{n}\right)\right\}$ is equivalent to $\left\{X_{1} \leq t_{1}, \ldots, X_{n} \leq t_{n}\right\}$. Hence we can transform $s \alpha \leq t$ in $s \alpha \leq t \theta_{[s, t]}$ (by applying $\theta_{[s, t]}$ to the tell part) and hence in a set of constraints in solved form $\left\{X_{1} \leq t_{1}, \ldots, X_{n} \leq t_{n}\right\}$ preserving the semantics. Moreover the evaluation of an element $Y \leq M \in A \cup B$ always succeeds. Therefore we can apply the substitution defined by the element to the remaining part of the sequence and hence delete such an element whitout affecting the semantics. The evaluation of an element $Y \leq m \in C \cup D$ always suspends. Therefore we can replace such an element by suspend (the evaluation of the whole $s \alpha \leq t \theta_{[s, t]}$ in this case can suspend or fail, depending on the other constraints).

2. If the atom $s \alpha \leq t \theta_{[s, t]}$ defines a constraint $X \leq t_{i}$ for a variable in $s \alpha$, (i.e. $X \leq t_{i} \in I_{1}$ ), we can replace each occurrence of $X$ by $t$ in the tell constraint without affecting the semantics.

3. The equivalence between tell (modified by the substitution obtained by the ask solved form computation) and $t e l l_{n}$ follows from the equivalence between a set of equations and its solved form $[21,16]$ and by noting that the equations of the set $F \cup G$ which are deleted from the tell solved form, do not affect the semantics of the answer according to definition 3.4 .

The following theorem shows that the solved form of equivalent ask : tell constraints is unique up to renaming. 
Theorem 4.20 Let $c_{1}=H_{1}:-a s k_{1}:$ tell $l_{1}$ and $c_{2}=H_{2}:-a s k_{2}:$ tell ${ }_{2}$ be equivalent according to definition 4.18. Let $c_{s o l_{1}}$ and $c_{s o l_{2}}$ be the solved forms of $c_{1}$ w.r.t. $\operatorname{Var}\left(H_{1}\right)$ and of $c_{2}$ w.r.t. $\operatorname{Var}\left(H_{2}\right)$ respectively. Then there exists a renaming $\rho$ such that $c_{\text {sol }}=c_{s o l_{2}} \rho$.

\section{Proof}

We can assume whitout loss of generality that $H_{1}=H_{2}=p\left(X_{1}, \ldots, X_{n}\right)$ (we can rename variables of $c_{i}, i \in\{1,2\}$, whitout affecting the semantics). We will show that if there exists no renaming $\rho$ such that $c_{s o l_{1}}=c_{s o l_{2}} \rho$, then $c_{s o l_{1}}$ and $c_{s o l_{2}}$ are not equivalent and then, for theorem 4.19, also $c_{1}$ and $c_{2}$ are not equivalent. Let us suppose that there exists no renaming $\rho$ such that $c_{\text {sol }}=c_{s_{2} l_{2}} \rho$. In the following cases

$$
\text { - } c_{n_{i}}=\text { fail and } c_{n_{j}} \neq \text { fail, }
$$

- suspend $\in c_{n_{i}}$ and suspend $\notin c_{n_{j}}$

$i, j \in\{1,2\}$ and $i \neq j, c_{s o l_{1}}$ and $c_{s o l_{2}}$ are not equivalent because of the intended meaning of suspend and fail. Then let us suppose that $c_{s o l_{1}}=p\left(X_{1}, \ldots, X_{n}\right):-a s k_{s o l_{1}}:$ tell $_{s o l_{1}}$ and $c_{s o l_{2}}=$ $p\left(X_{1}, \ldots, X_{n}\right):-a s k_{s o l_{2}}: t e l l_{s o l_{1}}$. If $X_{h} \leq t_{h} \in a s k_{s o l_{i}}$ and there exists no $X_{h} \leq l_{h} \in a s k_{s o l_{j}}$ ( $i$ and $j$ as before), then $c_{s o l_{1}}$ and $c_{s o l_{2}}$ are obviously not equivalent. Then let us suppose $a s k_{s o l_{1}}=$ $\left\{Y_{1} \leq t_{1}, \ldots, Y_{m} \leq t_{m}\right\}$ and $a s k_{s o l_{2}}=\left\{Y_{1} \leq l_{1}, \ldots, Y_{m} \leq l_{m}\right\}$, where $\left\{Y_{1}, \ldots, Y_{m}\right\} \subseteq\left\{X_{1}, \ldots, X_{n}\right\}$. If there exists no renaming $\rho$ such that $a s k_{s o l_{1}}=a s k_{s o l_{2}} \rho$ then there exists $h, 1 \leq h \leq m$, such that there exists no $\rho$ such that $t_{h}=l_{h} \rho$. Then $c_{s o l_{1}}$ and $c_{s o l_{2}}$ are not equivalent since one of the following two cases holds

1. $t_{h}$ and $l_{h}$ are not unifiable. Therefore given the initial state $\left\{Y_{1} \leq t_{1}, \ldots, Y_{m} \leq t_{m}\right\}$ the evaluation of $a s k_{s o l_{1}}$ succeeds while the evaluation of $a s k_{s o l_{2}}$ fails.

2. if $t_{h}$ and $l_{h}$ are unifiable (and $t_{h} \neq l_{h} \rho$ for any renaming $\rho$ ) one of the following subcases holds

a) $t_{h} \supseteq l_{h}\left(t_{h}\right.$ subsumes $l_{h}$, definition 4.4). Then given the initial state $\left\{Y_{1} \leq t_{1}, \ldots, Y_{m} \leq t_{m}\right\}$ the evaluation of $a s k_{s o l_{1}}$ succeeds, while the evaluation of $a s k_{s o l_{2}}$ suspends.

b) $l_{h} \supseteq t_{h}$. Then given the initial state $\left\{Y_{1} \leq l_{1}, \ldots, Y_{m} \leq l_{m}\right\}$ the evaluation of $a s k_{s o l_{2}}$ succeeds, while the evaluation of $a s k_{s o l_{1}}$ suspends.

c) $t_{h} \nsupseteq l_{h}$ and $t_{h} \nsupseteq l_{h}$. Then given the initial state $\left\{Y_{1} \leq t_{1}, \ldots, Y_{m} \leq t_{m}\right\}$ the evaluation of $a s k_{s o l_{1}}$ succeeds, while the evaluation of $a s k_{s a_{2}}$ suspends (and given the initial state $\left\{Y_{1} \leq l_{1}, \ldots, Y_{m} \leq l_{m}\right\}$ the evaluation of $a s k_{s o l_{2}}$ succeeds, while the evaluation of $a s k_{s o l_{1}}$ suspends).

Now let us suppose ther exists $\rho$ such that $a s k_{s o l_{1}}=a s k_{s o l_{2}} \rho$ and let us consider the tell constraints. Since $c_{\text {sol }_{2}}$ and $c_{s o l_{2}} \rho$ are equivalent we will consider $t e l l_{\text {sol }_{1}}$ and $t e l l_{\text {sol }_{2}} \rho$. We can assume that tell $_{\text {sol }}=\left\{Z_{1} \leq s_{1}, \ldots, Z_{k} \leq s_{k}\right\}$ and tell $_{\text {sol }_{2}} \rho=\left\{Z_{1} \leq f_{1}, \ldots, Z_{k} \leq f_{k}\right\}$ where $\left\{Z_{1}, \ldots, Z_{k}\right\} \subseteq W$, $W=\left\{X_{1}, \ldots, X_{n}\right\} \cup \operatorname{Var}\left(t_{1}, \ldots, t_{m}\right)$. In fact atoms which define bindings not relevant to semantics, i.e. atoms which define bindings $Z=t$ where $Z \notin W$, are deleted from the the solved form. Hence if $Z_{h} \leq s_{h} \in$ tell $_{\text {sol }_{1}}, 1 \leq h \leq k$ and there exists no $Z_{h} \leq f_{h} \in$ tell $_{\text {sol }_{2}}$, $c_{\text {sol }_{1}}$ and $c_{\text {sol }_{2}} \rho$ are not equivalent. As in the $a s k$ case, if there exists no renaming $\rho_{1}$ such that $t e l_{s o l_{1}}=t_{e l l_{s o l_{2}} \rho \rho_{1} \text {, then }}$ there exists $h, 1 \leq h \leq k$ such that there exists no renaming $\rho_{1}$ for which $s_{h}=f_{h} \rho_{1}$. Then given the initial state $\left\{Y_{1} \leq t_{1}, \ldots, Y_{m} \leq t_{m}\right\}$ (which allows to successfully evaluate the $a s k$ 's), according to definition 3.4 there exists an answer for $c_{s o l_{1}}$ which is not an answer for $c_{s o l_{2}} \rho$, because $s_{h}$ and $f_{h}$ are not variants. Therefore $c_{s o l_{1}}$ and $c_{s o_{2}} \rho$ are not equivalent and the thesis holds. 


\section{The solved form of reactive sequences and trees}

In this section we first give the definition of solved form for reactive sequences and reactive trees. Then we show an algorithm which computes the solved form of a reactive sequence and therefore of a reactive tree. Finally we prove the correctness of such an algorithm.

Definition 5.1 (solved form of reactive sequences) Let $s$ be the reactive sequence $H:-a s k_{1}:$ tell $_{1} \rightarrow a s k_{2}:$ tell $_{2} \rightarrow \ldots \rightarrow a s k_{n}:$ tell $_{n}$ and let $V_{1}=\operatorname{Var}(H), V_{i}=\operatorname{Var}\left(H:-a s k_{1}: \operatorname{tell}_{1} \rightarrow \ldots \rightarrow a s k_{i-1}: \operatorname{tell}_{i-1}\right), i=2, \ldots, n, W_{j}=$ $\operatorname{Var}\left(a s k_{j+1}:\right.$ tell $_{j+1} \rightarrow \ldots \rightarrow a s k_{n}:$ tell $\left._{n}\right), j=1, \ldots, n-1 . s$ is in solved form iff the following conditions hold

1. $a s k_{i}:$ tell $_{i}$ is in solved form w.r.t. $V_{i}$ and suspend $\notin a s k_{i}, i=1, \ldots, n-1$,

2. if $X \leq t \in a s k_{i}$ then $X \notin W_{i}$. If $X=t \in$ tell $_{i}$ then $X \notin W_{i}, i=1, \ldots, n-1$,

3. either $a s k_{n}:$ tell $_{n}$ is in solved form w.r.t. $V_{n}$ or $\Sigma\left(a s k_{n}\right) \cup$ tell $_{n}$ is not unifiable.

where $\Sigma(a s k)$ is defined as in definition 3.2 reactive tree $T$ is in solved form iff each reactive sequence in $T$ is in solved form.

\subsection{A solved form algorithm for reactive sequences and trees}

Let us now show the the $\mathcal{N}(s)$ procedure, which, given a reactive sequence $s$ returns an equivalent sequence $s_{\text {sol }}$ which is in solved form. The solved form of a reactive tree $\mathrm{T}$ can be obtained in a straightforward way by applying the $\mathcal{N}(s)$ procedure to each path (reactive sequence) in $\mathrm{T}$. The procedure is a direct extension of the solved form algorithm given in section 4.1 .

begin

Let $s$ be the reactive sequence $H:-a s k_{1}: \operatorname{tell}_{1} \rightarrow \ldots \rightarrow a s k_{m}: t e l l_{m}$. Let $s_{\text {nor }}=H:-a s k_{n_{1}}$ : tell $l_{n_{1}} \rightarrow \ldots \rightarrow a s k_{n_{i}}: t e l l_{n_{i}}$ be the sequence resulting from the solved form of $H:-a s k_{1}: t e l l \rightarrow$ $\ldots \rightarrow a s k_{i}: t_{e l l}$ and let $V_{i}=\operatorname{Var}\left(s_{n_{n r_{i}}}\right)$. Let $s_{\gamma_{e m_{i}}}=a s k_{r_{i+1}}: t e l l_{r_{i+1}} \rightarrow \ldots \rightarrow a s k_{r_{m}}: t e l l_{r_{m}}$ be the (part of the) sequence to be reduced after the solved form computation of $H:-a s k_{1}:$ tell $_{1} \rightarrow \ldots \rightarrow$ $a s k_{i}:$ tell $_{i}$. Thus $s_{\text {nor }}=H:-\lambda$ (empty sequence) and $s_{r e m_{0}}=a s k_{1}:$ tell $_{1} \rightarrow \ldots \rightarrow a s k_{m}:$ tell $_{m}$. Let $a s k_{n_{i}}: t e l l_{n_{i}}$ be the result of the solved form of $a s k_{r_{i}}: t e l l_{r_{i}}$ w.r.t. the set of variables $V_{i}$ as computed by the algorithm of section 4.1 . Moreover let $\theta_{[s, t]}$, alpha, $F$ and $G$ as resulting by the definitions in that algorithm. Then

i) (failure) If $a s k_{n_{i}}:$ tell $_{n_{i}}=$ fail then STOP and $\mathcal{N}(s)=s_{n_{0 r_{i-1}}} \rightarrow$ fail.

ii) (suspension) If suspend $\in a s k_{n_{i}}$ then STOP and $\mathcal{N}(s)=s_{n o r_{i-1}} \rightarrow a s k_{n_{i}}:$ tell $_{n_{i}}$.

iii) (success) If $i=m$ then $\mathcal{N}(s)=s_{n_{0} r_{i-1}} \rightarrow a s k_{n_{i}}: t_{t} l_{n_{i}}$. If $i<m$ then

- $s_{\text {nor }_{i}}=s_{\text {nor }_{i-1}} \rightarrow a s k_{n_{i}}:$ tell $_{n_{i}}$,

- Let $s_{r e m_{i-1}}=\left(a s k_{r_{i}}: t_{e l l} l_{r_{i}} \rightarrow \ldots \rightarrow a s k_{r_{n}}:\right.$ tell $\left._{r_{n}}\right)$. Then we define the part of the sequence to be reduced at the next step $s_{r e m_{i}}$ as follows

$$
\begin{aligned}
& s_{\text {remi }}^{1}=s_{r e m_{i-1}}^{1} \theta_{[s, t]}=\left(a s k_{r_{i+1}}: \text { tell }_{r_{i+1}} \rightarrow \ldots \rightarrow a s k_{r_{n}}: \text { tell }_{r_{n}}\right) \theta_{[s, t]}, \\
& s_{\text {remi }}^{2}=s_{\text {rem }}^{1} \alpha, \\
& s_{\text {remi }}=s_{\text {remi }}^{2} \gamma, \text { where } \gamma=\left\{X / t \mid(X=t) \in \text { tell }_{n_{i}} \cup F\right\} \cup\{Y|X| X=Y \in G\} .
\end{aligned}
$$

end.

The bindings defined by the solved form computation of $a s k_{r_{i}}: t e l l_{r_{i}}$ have to be passed to the remaining part of the sequence in order to get the solved form preserving the semantics. Hence 
$s_{\text {rem }}$ is defined as above. Moreover if the solved form $a s k_{n_{i}}: t_{e l l_{n_{i}}}$ either contains suspend or fail, the procedure stops deleting the remaining part $s_{\text {remi }}$ from the solved form. This does not affect the semantics since such a part would never be evaluated. Let us show an example of the solved form of a reactive tree.

\section{Example 5.2}

$$
\text { T1) } \begin{aligned}
q(X, Y): & -\{f(X) \leq f(g(Z))\}:\{Y=X\} \rightarrow \\
& ((\{Y=g(g(W))\}:\{X=g(g(b))\})+(\{Y=g(W)\}:\{W=a, Z=b\})) .
\end{aligned}
$$

The following tree $T_{2}$ is the solved form of the previous tree $T_{1} . T_{1}$ and $T_{2}$ are semantically equivalent reactive trees

$$
\text { T2) } \begin{aligned}
q(X, Y): & -\{X \leq g(Z)\}:\{Y=g(Z)\} \rightarrow \\
& ((\{Z \leq g(W)\}:\{W=b)\})+(\{\}:\{\text { fail }\})) .
\end{aligned}
$$

\subsection{The equivalence between a reactive tree and its solved form}

The following lemma shows that after the application of one step of the previous procedure to a solved sequence $s$, the resulting sequence is still a reactive sequence (definition 5.1 ). Then the procedure is well defined.

\section{Lemma 5.3 Let $s$ be the reactive sequence}

$H:-a s k_{1}:$ tell $_{1} \rightarrow \ldots \rightarrow a s k_{m}:$ tell $_{m_{m}}$. Let ask $k_{n_{1}}:$ tell $_{n_{2}}$ be the element resulting from the normalization of ask $k_{1}:$ tell $l_{1}$. Let $s_{r_{e m_{2}}}=a s k_{r_{2}}:$ tell $_{r_{2}} \rightarrow \ldots \rightarrow a s k_{r_{n}}:$ tell $r_{r_{n}}$ be defined as in the previous procedure. Then $p\left(X_{1}, \ldots, X_{n}\right):-a s k_{n_{1}}:$ tell $_{n_{1}} \rightarrow s_{\text {rem }}$ is a reactive sequence as defined in definition 5.1 .

\section{Proof}

$p\left(X_{1}, \ldots, X_{n}\right):-a s k_{n_{1}}: t e l l_{n_{1}}$ satisfies the conditions of definition 5.1 by the definition of the procedure. Let $\theta$ be the substitution obtained from the computation of the solved form of $a s k_{1}$ : tell $_{1}$ which is applied to the remaining part of the sequence $s$. Let $a s k_{i}=\left\{s_{i_{1}} \leq t_{i_{1}}, \ldots s_{i_{m(i)}} \leq\right.$ $\left.\left.t_{i_{m(i)}}\right\}, D=\operatorname{Dom}(\theta) \cup \operatorname{Cod}(\theta)\right\}, V_{i}=\operatorname{Var}\left(H:-a s k_{1}: \operatorname{tell}_{1} \rightarrow \ldots \rightarrow a s k_{i-1}: \operatorname{tell}_{i-1}\right), L_{i}=$ $\operatorname{Var}\left(s_{i_{1}}, \ldots, s_{i_{m(i)}}\right), R_{i}=\operatorname{Var}\left(t_{i_{1}}, \ldots, t_{i_{m(i)}}\right), N e w=\left\{X \mid X \notin V_{n+1}\right\}$. By hypothesis $s$ is a reactive sequence, hence $V_{i} \cap R_{i}=\emptyset, L_{i} \subseteq V_{i}$ and $L_{i} \cap R_{i}=\emptyset, 1 \leq i \leq n$. Let $\operatorname{Var}\left(R_{j} \theta\right)=\operatorname{Var}\left(t_{j_{1}} \theta, \ldots \leq\right.$ $\left.t_{j_{m(j)}} \theta\right)$, and let $\operatorname{Var}\left(L_{j} \theta\right)$ and $\operatorname{Var}\left(V_{j} \theta\right)$ be analogously defined. Then by definiton of $\mathcal{N}(s)$ we have that $D \subseteq V_{2} \cup N e w$. Therefore $D \cap R_{j}=\emptyset$ and hence $\operatorname{Var}\left(R_{j} \theta\right)=R_{j}$ for $j=2, \ldots, n$. Then for $j=2, \ldots n, \operatorname{Var}\left(L_{j} \theta\right) \cup \operatorname{Var}\left(R_{j} \theta\right)=\emptyset$ and $\operatorname{Var}\left(V_{i} \theta\right) \cup \operatorname{Var}\left(R_{j} \theta\right)=\emptyset$ and the thesis holds.

It is straightforward to show that the sequence $s_{s o l}$ obtained by $\mathcal{N}(s)$ is a sequence in solved form. The following lemma is an obvious consequence of the definition of $\mathcal{N}(s)$.

Lemma 5.4 Let $s$ be a reactive sequence. Then $s_{\text {sol }}=\mathcal{N}(S)$ is in solved form.

Let us now show the equivalence between a reactive sequence and its solved form according to the semantics of reactive trees (and sequences) given in section 3.1 .

Theorem 5.5 Let $s=H:-a s k_{1}:$ tell $_{1} \rightarrow \ldots \rightarrow a s k_{m}:$ tell $_{m}$ be a reactive sequence and let $s_{\text {sol }}$ $=\mathcal{N}(s)=H:-a s k_{n_{1}}:$ tell $_{n_{1}} \rightarrow \ldots \rightarrow a s k_{n_{m}}:$ tell $_{n_{m}}$. Then $s$ and $s_{\text {sol }}$ are equivalent, i.e. given an initial state $\sigma_{i n}$, the evaluation of $s$ yields the answer $\langle\Phi, \sigma\rangle(\Phi \in\{$ success, fail, deadlock\}) (definition 3.4) iff the evaluation of $s_{\text {sol }}$ yields the answer $\left\langle\Phi^{\prime}, \sigma^{\prime}\right\rangle$ with $\Phi=\Phi^{\prime}$ and $\theta=\theta^{\prime} \rho$, where $\theta$ is an idempotent mgu of $\sigma, \theta^{\prime}$ is an idempotent $m g u$ of $\sigma^{\prime}$ and $\rho$ is a renaming. 


\section{Proof}

The proof can be carried out by induction on the number of steps performed to compute the solved form of the sequence. For the first step we can repeat the proof in theorem 4.19 to show that the semantic is preserved for the the solved form $a s k_{n_{1}}: t e l l_{n_{1}}$ of the first constraint. Moreover the semantics is preserved also for the remaining part of the sequence modified by the application of the substitutions $\theta_{[s, t]}, \alpha$ and $\gamma$, since those substitutions performs only bindings defined by $a s k_{n_{1}}:$ tell $_{n_{1}}$. Let $s_{i-1}=H:=a s k_{n_{1}}:$ tell $_{n_{1}} \rightarrow \ldots \rightarrow a s k_{n_{i-1}}:$ tell $_{n_{i-1}} \rightarrow a s k_{r_{i}}:$ tell $_{r_{i}} \rightarrow \ldots \rightarrow$ $a s k_{r_{n}}:$ tell $_{r_{\mathrm{n}}}$ be the sequence obtained by the transformation to the first $i-1$ atoms (i.e. the $a s k_{n_{j}}: t e l l_{n_{j}}, j=1, \ldots n$ are constraints in solved form. Let us suppose by inductive hypothesis that the original sequence $s$ and $s_{i-1}$ are equivalent. To show the equivalence between $s_{i-1}$ and $s_{i}$ we can repeat the same argument of the base step, the solved form computation of $\left(a s k_{r_{i}}: t e l l_{r_{i}}\right) \alpha_{n_{\text {nor }}}$ where the substitution defining the initial state is given by $\alpha_{\text {nor }}$ which is an idempotent mgu of the state obtained by the (successful) evaluation of $H \alpha_{1}:-a s k_{n_{1}}: \operatorname{tell}_{n_{1}}, \ldots, a s k_{n_{i-1}}: \operatorname{tell}_{n_{i-1}}\left(\alpha_{1}\right.$ defines the original initial state). We can apply also in this case lemma 4.17 because by lemma 5.3 and by the definition of reactive sequence, (if $\alpha_{1}$ satisfies the hypothesis of the lemma 4.17 ) also $\alpha_{\text {nori }}$ satisfies the hypothesis of the lemma 4.17 .

Finally, the following theorem shows the equivalence between a reactive tree and its solved form.

Theorem 5.6 Let $T$ be a reactive tree and let $T_{\text {sol }}$ be the solved form of $T$. Then $T$ and $T_{\text {sol }}$ are equivalent, i.e. given a state $\sigma_{\text {in }},\langle\Phi, \sigma\rangle$ is an answer for $T$ with initial state $\sigma_{\text {in }}$ (definition 3.5) iff $\left\langle\Phi^{\prime}, \sigma^{\prime}\right\rangle$ is an answer for $T_{\text {sol }}$ with initial state $\sigma_{i n}$, where $\Phi=\Phi^{\prime}, \theta=\theta^{\prime} \rho, \theta$ is an idempotent mgu of $\sigma, \theta^{\prime}$ is an idempotent mgu of $\sigma^{\prime}$ and $\rho$ is a renaming.

Proof

Straightforward by theorem 5.5 and by definition 3.5 .

\section{Future work}

The solved form of a reactive tree $T$ can replace $T$ in any context, thus performing a sort of pre-compilation of concurrent constraint processes represented by trees. At run time, the process may be embedded in a set of other processes, which may affect its behaviour. According to the result of theorem 5.6, the transformation to the solved form is valid with respect to any context, i.e. does not modify the process (partial) computations and therefore does not change the possible inter-process data comunications. Then such a pre-compilation does not modify the semantics of the whole set of concurrent constraint processes. This work can be extended in various interesting directions. For example, the constraint system can be changed to a richer one, for which there exists a solved form in the pure constraint case. Another interesting problem is the definition of stronger equivalence relations on reactive trees. For example, the construction of [25] which uses a bisimulation on tree structures similar to ours, can easily be adapted to the case of reactive trees in solved form. Finally, it is possible to view tree expressions occurring in reactive trees as constraints with their own notions of solutions and consistency and their transformations to solved form.

\section{References}

[1] K.R. Apt. Introduction to Logic Programming. In J. van Leeuwen, editor, Handbook of Theoretical Computer Science, volume B: Formal Models and Semantics. Elsevier, Amsterdam and The MIT Press, Cambridge, 1990. 
[2] K.L. Clark and S. Gregory. PARLOG: parallel programming in logic. ACM Transactions on Programming Languages and Systems, 8:1-49, 1986.

[3] H. Comon and P. Lescanne. Equational Problems and Disunification. Journal of Symbolic Computation, $7: 371-425,1989$.

[4] F.S. de Boer, J.N. Kok, C. Palamidessi, and J.J.M.M. Rutten. Semantic models for a version of PARLOG. In G. Levi and M. Martelli, editors, Proc. Sixth Int'l Conf. on Logic Programming, pages 621-636. The MIT Press, Cambridge, Mass., 1989. Extended version to appear in Theoretical Computer Science.

[5] F.S. de Boer and C. Palamidessi. Concurrent logic languages: Asynchronism and language comparison. In S. Debray and M. Hermenegildo, editors, Proc. North American Conf. on Logic Programming'90, pages 99-114. The MIT Press, Cambridge, Mass., 1990.

[6] F.S. de Boer and C. Palamidessi. A Fully Abstract Model of Concurrent Logic Languages. Technical report, Dipartimento di Informatica, Università di Pisa, 1990.

[7] F.S. de Boer and C. Palamidessi. On the asynchronous nature of communication in concurrent logic languages: A fully abstract model based on sequences. In J.C.M. Baeten and J.W. Klop, editors, Proc. of Concur 90, volume 458 of Lecture Notes in Computer Science, pages 175-194. Springer-Verlag, Berlin, 1990.

[8] M. Falaschi, M. Gabbrielli, G. Levi, and M. Murakami. Nested Guarded Horn Clauses: a language provided with a complete set of Unfolding Rules. International Journal on Foundations of Computer Science, 1990. to appear.

[9] M. Gabbrielli and G. Levi. Modeling answer constraints in Constraint Logic Programs. Technical report, Dipartimento di Informatica, Università di Pisa, 1990.

[10] M. Gabbrielli and G. Levi. Unfolding and Fixpoint Semantics of Concurrent Constraint Programs. In H. Kirchner and W. Wechler, editors, Proc. Second Int'l Conf. on Algebraic and Logic Programming, volume 463 of Lecture Notes in Computer Science, pages 204-216. Springer-Verlag, Berlin, 1990.

[11] H. Gaifman, M.J. Maher, and E.Y. Shapiro. Reactive Behavior Semantics for Concurrent Constraint Logic Programs. In E. Lusk and R. Overbeck, editors, Proc. North American Conf. on Logic Programming'89, pages 553-572. The MIT Press, Cambridge, Mass., 1989.

[12] S. Gregory. Parallel Logic Programming in PARLOG: the Language and its Implementation. Addison-Wesley, 1987.

[13] J. Jaffar and J.-L. Lassez. Constraint Logic Programming. In Proc. Fourteenth Annual ACM Symp. on Principles of Programming Languages, pages 111-119. ACM, 1987.

[14] J. Jaffar and J.-L. Lassez. Constraint Logic Programming. Technical report, Department of Computer Science, Monash University, June 1986.

[15] C. Kirchner and P. Lescanne. Solving Disequations. In Proc. Second IEEE Symp. on Logic In Computer Science, pages 347-352. IEEE Computer Society Press, 1987.

[16] J.-L. Lassez, M.J. Maher, and K. Marriott. Unification Revisited. In J. Minker, editor, Foundations of Deductive Databases and Logic Programming, pages 587-625. Morgan Kaufmann, Los Altos, Ca., 1988. 
[17] J.L. Lassez and K. McAloon. Applications of a canonical form for generalized linear constraints. In Proc. Int'l Conf. on Fifth Generation Computer Systems, pages 703-710, Tokyo, 1988. Institute for New Generation Computer Technology.

[18] G. Levi. Models, Unfolding Rules and Fixpoint Semantics. In R.A. Kowalski and K.A. Bowen, editors, Proc. Fifth Int'l Conf. on Logic Programming, pages 1649-1665. The MIT Press, Cambridge, Mass., 1988.

[19] J.W. Lloyd. Foundations of logic programming. Springer-Verlag, Berlin, 1987. second edition.

[20] M.J. Maher. Logic semantics for a class of committed-choice programs. In J.-L. Lassez, editor, Proc. Fourth Int'l Conf. on Logic Programming, pages 858-876. The MIT Press, Cambridge, Mass., 1987.

[21] A. Martelli and U. Montanari. An efficient unification algorithm. ACM Transactions on Programming Languages and Systems, 4:258-282, 1982.

[22] D.S. Parker and R.R. Muntz. A theory of directed logic programs and streams. In R.A. Kowalski and K.A. Bowen, editors, Proc. Fifth Int'l Conf. on Logic Programming, pages 620650. The MIT Press, Cambridge, Mass., 1988.

[23] V.A. Saraswat. Partial Correctness Semantics for $\mathrm{CP}(\emptyset, 1, \&)$. In Proc. of the Conf. on Foundations of Software Computing and Theoretical Computer Science, volume 206 of Lecture Notes in Computer Science, pages 347-368. Springer-Verlag, Berlin, 1985.

[24] V.A. Saraswat. Concurrent Constraint Programming Languages. PhD thesis, Carnegie-Mellon University, January 1989.

[25] V.A. Saraswat and M. Rinard. Concurrent constraint programming. In Proc. Seventeenth Annual ACM Symp. on Principles of Programming Languages. ACM, 1990.

[26] E.Y. Shapiro. Concurrent Prolog: Collected Papers. The MIT Press, Cambridge, Mass., 1988. 